\title{
The extracorporeal circulation with transdiaphragmatic approach in living-donor liver transplantation for hepatoblastoma with atrial extension of tumor thrombus
}

Mureo Kasahara, Seisuke Sakamoto, Yusuke Yanagi, Hajime Uchida, Seiichi Shimizu, Kotaro Mimori, Yasuyuki Kameoka, Akinari Fukuda

Department of Organ Transplantation, National Center for Child Health and Development, Tokyo, Japan

Background: Surgical intervention for hepatoblastoma (HB) with tumor thrombi extending into the inferior vena cava (IVC) and the right atrium (RA) might require careful planning of the surgical procedures, including vascular reconstruction and extracorporeal circulation.

Methods: We herein report a successful case of living-donor liver transplantation (LDLT) for HB with atrial extension of a tumor thrombus by extracorporeal circulation with a transdiaphragmatic approach.

Results: The patient was a 5-year-old boy with PRETEXT IV HB with a tumor thrombus that extended into the IVC and the RA. After four cycles of chemotherapy (PHITT group D: high-risk protocol) and resection of bilateral lung metastases, the size of the primary HB tumor decreased. As the tumor extension from the left hepatic vein to the RA had decreased but was still present, we performed LDLT with tumor thrombectomy. The central part of the diaphragm was sagittally incised to expose the suprahepatic IVC and the RA. Venovenous bypass was achieved from the right femoral vein and inferior mesenteric vein to the RA. En bloc resection of the native liver with the tumor thrombus was then performed. Hepatic vein anastomosis was made between the newly created orifice on the IVC and the graft left hepatic vein. The duration of LDLT was 10 hours and 44 minutes (extracorporeal circulation time, 78 minutes).

Conclusions: Pediatric liver transplantation for HB with the extension of tumor thrombi into the RA under extracorporeal circulation is a feasible option and allows for the expansion of the indications for transplantation for children with unresectable liver tumors.

Corresponding author: Mureo Kasahara

E-mail: kasahara-m@ncchd.go.jp

(c) The Korean Society for Transplantation

This is an Open Access article distributed under the terms of the Creative Commons Attribution Non-Commercial License (http://creativecommons.org/licenses/by-nc/4.0/) which permits unrestricted non-commercial use, distribution, and reproduction in any medium, provided the original work is properly cited. 